\title{
Model for Dynamic Multiple of CPPI Strategy
}

\author{
Guangyuan Xing, ${ }^{1}$ Yong Xue, ${ }^{2}$ Zongxian Feng, ${ }^{1}$ and Xiaokang $\mathrm{Wu}^{3}$ \\ ${ }^{1}$ School of Finance and Economics, Xi'an Jiaotong University, Xi'an 710061, China \\ ${ }^{2}$ Financial Market Department, Bank of Xi'an, Xi'an 710075, China \\ ${ }^{3}$ School of Management, Xi'an Jiaotong University, Xian 710049, China
}

Correspondence should be addressed to Zongxian Feng; zxfeng@mail.xjtu.edu.cn

Received 13 March 2014; Accepted 28 May 2014; Published 24 June 2014

Academic Editor: Chuangxia Huang

Copyright (C) 2014 Guangyuan Xing et al. This is an open access article distributed under the Creative Commons Attribution License, which permits unrestricted use, distribution, and reproduction in any medium, provided the original work is properly cited.

Focusing on the parameter "Multiple" of CPPI strategy, this study proposes a dynamic setting model of multiple for gap risk management purpose. First, CPPI gap risk is measured as the probability that the value loss of active asset exceeds its allowed maximum drop determined by a given multiple setting. Moreover, according to the statistical estimation using SV-EVT approach, a dynamic choice of multiple is detailed as a function of time-varying asset volatility, expected loss, and the possibility of occurrence of extreme events in the active asset returns illustrated empirically on Shanghai composite index data. This study not only enriches the literature of dynamic proportion portfolio insurance, but also provides a practical reference for CPPI investors to choose a moderate risky exposure achieving gap risk management, which promotes CPPI's application in emerging capital market.

\section{Introduction}

Portfolio insurance strategy can help investors control downside risk of asset value on retention of upward market opportunities; it mainly includes dynamic strategies like OBPI based on replication options, CPPI and TIPP based on parameter setting, and static strategies like stop-loss and buy-hold, in which CPPI (constant proportion portfolio insurance) strategy is based on parameter setting. Black and Jones [1] described basic execution procedure as follows: the initial investment volume is $V_{0}$, the period of ensuring not to lose capital investment is $T$, terminal guaranteed value $G$ is proportional to the initial investment volume $V_{0}, G=\lambda V_{0}$, and the required terminal asset value $V_{T} \geq G$. In CPPI strategy, we define the bottom-line value of the asset portfolio $F_{t}=G \cdot e^{-r(T-t)}(0 \leq t \leq T)$ in every moment. $r$ is riskfree interest rate; the difference between portfolio value and bottom-line value is a cushion; $C_{t}=V_{t}-F_{t}$. We set the multiple and multiply it by the cushion, invest the amount of capital on risky assets and the remainder on risk-free assets, dynamically allocate the risky and risk-free assets in the time horizon, and achieve the goal of portfolio insurance. CPPI strategy which is initially put forward by Black and Jones shows considerable simplicity and flexibility compared with other portfolio insurance strategies; for example, with no maturity date limit, risk exposure can be chosen according to risk appetite of investors. Meanwhile, CCPPI strategy has solid theoretical foundation. Kingston [2] demonstrates that CPPI strategy is optimal when and only when the investors have decreasing absolute and relative risk aversion. Black and Perold [3] studied the impact of transaction cost and borrowing constraints on portfolio insurance strategy under CPPI strategy. They find that when there is no transaction cost, CPPI strategy is equivalent to permanent American buy right investment, and piecewise HARA utility function is optimal under the restriction of minimum consumption. With the increasing of the multiple, the payoffs of CPPI strategy are close to those of stop-loss strategy; the relationship between expected payoffs in holding period and multiplier is monotonous; expected payoffs of CPPI strategy are larger than those of stop-loss strategy [1-3]. Different from portfolio insurance strategy based on option replication, which needs complicated option pricing technology, CPPI strategy is much easier in operation because it is only based on parameter setting. Therefore, it is widely used as an important investment technology in guaranteed fund [4]. All 
the guaranteed funds in China have been using CPPI strategy or other investment strategies with CPPI strategy at the core since southern safe-haven growth fund, the first guaranteed fund, came into existence.

The setting of multiple (denoted by $m$ in the following model) is crucial in the operation of CPPI strategy, since it directly determines the risk exposure. The bigger the multiple $m$, the stronger the portfolio participates in the growing market. But it is also accompanied by much bigger risks. When the risky assets fall, the portfolio value falls quickly. Thus the research on CPPI strategy mainly focused on the setting of the multiple $m$. In traditional CPPI strategy, $m$ was preestablished as a fixed value, and it never changes with the market conditions. Some scholars make an improvement by putting forward variable ratio portfolio insurance strategy, which means dynamic parameter settings in CPPI strategy.

Chen et al. believe that the fixed multiple in CPPI strategy should rectify with the market conditions. They put forth dynamic constant proportion portfolio insurance (DPPI) by using genetic algorithm considering several factors related to the market volatility. The empirical results show that DPPI strategy is more profitable than CPPI strategy [5]. Chen and Liao believe that the investor has his implicit or explicit goal in an investment; thus they proposed goaldirected strategy to describe the trading behavior of investors which, being integrated with CPPI strategy, got a staged goal-directed CPPI strategy (GDCPPI) and was further extended into staged nonlinear goal-directed CPPI strategy [6]. Lee et al. proposed variable proportion portfolio insurance (VPPI) strategy, which enlarges or lessens the multiple when share price rises or falls. They believe portfolio insurance strategy based on this principle will produce better performance [7].

Different multiple dynamic setting principles make the CPPI strategy more profitable or more in line with the investor' targets. Unfortunately, the previous study ignored a real problem in operation: the continuous adjustment of assets in theory leads to some gap risks. Under continuous time frame, CPPI portfolio value at any moment is shown in formula (1) [3], in which $V_{t}$ is portfolio value at $t ; F_{t}$ is bottomline value at $t ; S_{t}$ is risky assets value at $t ; m$ is multiple in CPPI strategy; $r$ is risk-free interest rate; $\sigma$ is volatility of risk assets value; $0 \leq t \leq T ; V_{0}, F_{0}$, and $S_{0}$ denote initial values $(t=0)$ of variables. $V_{t}$ is not less than $F_{t}$ regardless of $m$, and terminal guaranteed value can always be achieved. It suggests that when setting the multiple in the research on DPPI, we need only to take into consideration the risk preference and expected return rate of investors:

$$
\begin{aligned}
V_{t}= & F_{t}+\left(V_{0}-F_{0}\right)\left(\frac{S_{t}}{S_{0}}\right)^{m} \\
& \times \exp \left\{\left(r-m\left(r-\frac{\sigma^{2}}{2}\right)-\frac{1}{2} m^{2} \sigma^{2}\right) t\right\} .
\end{aligned}
$$

In the real market conditions, because of the existence of market friction factors like transaction cost, rebalancing happens on the discrete adjustment point. It is possible that portfolio value drops below bottom-line value because of the

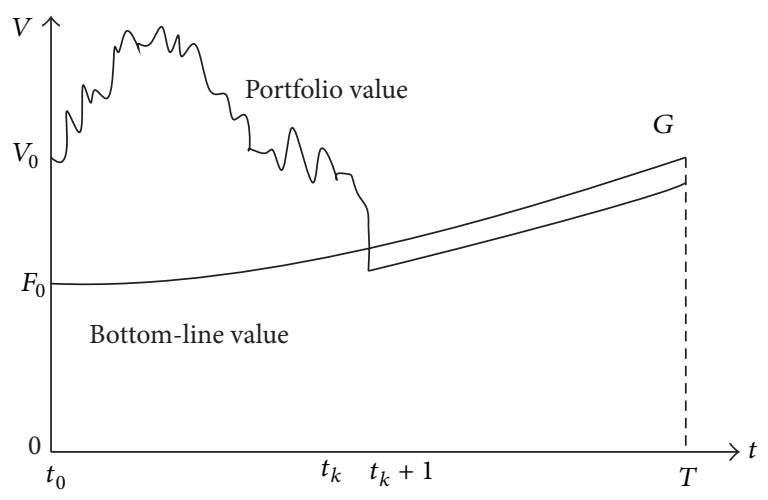

Figure 1: Gap risk of CPPI.

sharp fall of risky assets value between two adjustment points, facing the risk that CPPI portfolio value cannot achieve its guaranteed value on the due day. Figure 1 shows the situation in which gap risk happens between two adjustment points $t_{k}$ and $t_{k+1}$ possibly because risky assets value falls sharply before CPPI investors rebalance assets. Meanwhile, CPPI portfolio value $V_{t}$ falls under bottom-line value $F_{t}$. The whole funds can only be invested on risk-free assets.

Therefore, Balder et al. defined discrete CPPI strategy and its gap risk which is more congruent to the market environment [8]. In terms of the management of gap risk in CPPI strategy, Cont and Tankov [9] studied the situation of downward jump of object portfolio and its CPPI strategy, the possibility of portfolio value reaching the bottom-line value, the expected loss and distribution of the loss, and the measure of gap risk they took to study the problem of hedging the gap risk in CPPI strategy by option. Considering transaction cost and borrowing constraints, Jessen studied CPPI strategy under the condition of discrete transactions. He believed that gap risk can be avoided by charging, hedging, or setting a "mendacious" guaranteed value, but the latter two cost less for investors [10].

Although the study above considered gap risk in CPPI strategy, it mainly concentrated on extracting the administrative cost and managing the gap risk by option hedging without any attention to the setting of the multiple. In this paper, based on the discrete CPPI strategy by Balder et al. [8], we built a dynamic multiple setting model according to the estimation of extreme changes in risky assets, aiming at studying gap risk management in CPPI strategy from "dynamic proportion." Different from mature capital markets, financial derivatives and their trading are not that abundant in China or in other developing countries. No financial products can be used directly to hedge gap risks in CPPI strategy; thus a multiple setting perspective is needed to handle gap risks. No research has been done on how to dynamically set the multiple in order to avoid gap risks and improve the venture capital in fluctuating market environment. Additionally, dynamic multiple setting model in this paper enriched theoretical system in dynamic proportion portfolio insurance strategy. The basis of dynamic multiple setting is given from the gap risk management perspective, 
which reveals that multiple setting is not simply to enlarge or lessen the multiple according to the rise or fall of share price [7] but is a complex decision based on risky assets value fluctuation, the frequency of occurrence of extreme prices, and expected loss. Since the dynamic multiple setting provides a basis for gap risk management, the conclusion in this paper offered upper limit of multiple setting in CPPI strategy; further research may consider investment preference and object of investors under this constraint.

\section{Model}

2.1. Gap Risks and Modeling Basis. In discrete CPPI strategy, asset allocation happens on equally spaced time series [8], defined as $\left\{t_{0}^{n}=0<t_{1}^{n} \cdots<t_{k}^{n}<t_{k+1}^{n} \cdots<t_{n}^{n}=\right.$ $\left.T, t_{k+1}^{n}-t_{k}^{n}=T / n\right\}$, where $n$ denotes times number of assets allocation in break-even period. In the practice, it has always been adjusted week by week according to the fixed cycle adjustment principle; since risky assets equal to the multiple $m$ multiply the cushion in CPPI strategy, the largest tolerable drop of risky assets value is $1 / m$. If risky assets value drop is $1 / m$ between two discrete adjustment points $t_{k}$ and $t_{k+1}, r$ is risk-free interest rate between $t_{k}$ and $t_{k+1}$, as shown in

$$
\begin{aligned}
V_{t_{k+1}} & =m C_{t_{k}}\left(1-\frac{1}{m}\right)+\left(V_{t_{k}}-m C_{t_{k}}\right)(1+r) \\
& =\left(V_{t_{k}}-C_{t_{k}}\right)+\left(V_{t_{k}}-m C_{t_{k}}\right) r \\
& =F_{t_{k}}+\left(V_{t_{k}}-m C_{t_{k}}\right) r \\
& \leq F_{t_{k+1}}
\end{aligned}
$$

Portfolio value $V_{t_{k+1}}$ will be less than or equal to bottomline value $F_{t_{k+1}}$ at $t_{k+1}$, and the whole CPPI portfolio assets invested in risk-free assets increase with risk-free interest rate. Therefore, $1 / m$ provides the largest drop of risky assets in an adjustment period $\left(t_{k}, t_{k+1}\right)$; we take the probability of risky assets value loss more than $1 / m$ in adjustment period as a measurement of gap risks; the probability of risky assets value loss more than $1 / m$ should be very small when setting $m$ in the management of this risk. The measurement of gap risk is shown in

$$
P\left(x_{k}>\frac{1}{m}\right)=\alpha
$$

where $x_{k}$ denotes risky assets value loss in adjustment period $\left(t_{k}, t_{k+1}\right)$ and $\alpha$ denotes the probability of risky assets value loss more than $1 / \mathrm{m}$. Formula (3) demonstrates the relationship between multiple and the change of risky assets value under the requirement of gap risk management; $\alpha$ is given based on the requirement of CPPI investors. The smaller $\alpha$ means a stronger risk control requirements. In this paper, $\alpha=0.1 \%$. Multiple setting based on the probability of gap risk offers an upper limit of the multiple for CPPI investors. In this way, we increased risky assets investment under the premise of gap risk avoiding and thus a higher expected value.
2.2. Multiple Dynamic Setting Model in CPPI Strategy. Due to the constant fluctuations in the value of assets, dynamic multiplier set is needed to meet the requirements of formula (3). Firstly, we must describe the characteristics of the movements of the risk asset value. Based on the SV-EVT description of the extreme price behavior of the market, this paper gives model to dynamically set the multiplier. Against the price fluctuations and volatility clustering time-varying of the risk asset, the autoregressive conditional heteroscedasticity (ARCH) models $[11,12]$ and stochastic volatility (SV) model [13] are applicative. ARCH model introduces conditional variance to analyze the variance variability and the fluctuation in the course is a linear function of the past observations and the square of the hysteretic disturbance. But in view of the financial time series "fat tail," the ARCH model seems fragile with the weak leverage and lasting square sequence [14]. Besides, the SV model is considered more suitable for describing the characteristics of the actual financial market volatility because the fluctuation is decided by a random process. Therefore, this paper uses the SV model to describe the risk assets yield characteristics with the following form:

$$
\begin{gathered}
x_{k}=\mu+\sigma_{k} z_{k}, \quad z_{k} \sim \text { i.i.d } \\
\ln \sigma_{k}^{2}=v+\phi\left(\ln \sigma_{k-1}^{2}-v\right)+\eta_{k}, \quad \eta_{k} \sim \text { i.i.d } N\left(0, \tau^{2}\right),
\end{gathered}
$$

where $x_{k}$ denotes risky assets value loss in adjustment period $\left(t_{k}, t_{k+1}\right)$, the loss can be defined as negative return, and $x_{k}=-\left(p_{t_{k+1}}-p_{t_{k}}\right) / p_{t_{k}}$. Corresponding to formula (2), we use hundred yields. $p_{t_{k+1}}$ and $p_{t_{k}}$ are assets value in adjustment time point; $\mu$ denotes expected loss of risky assets; $z_{k}$ denotes residual items of independent identically distribution; $\sigma_{k}$ denotes potential fluctuations; $v$ is constant terms of wave equation, and it denotes mean value of logarithmic fluctuations; $\eta_{k}$ denotes wave disturbance level of independent identically distribution, which follows normal distribution with 0 mean and $\tau^{2}$ variance; error term $\eta_{k}$ and $z_{k}$ are mutually independent; $\phi$ is continuous parameter, reflecting the influence of current fluctuation on the future, for $|\phi|<1$; SV model is covariance stationary.

According to formula (3), we control gap risk less than $\alpha$, namely, guarantee portfolio insurance with confidence level $1-\alpha$, equivalent to

$$
P\left\{x_{k}<\frac{1}{m_{k}}\right\}=\int_{-\infty}^{1 / m_{k}} f\left(x_{k}\right) d x_{k}=1-\alpha,
$$

where $f\left(x_{k}\right)$ is probability density function of loss $x_{k}$; the standard form of the above Formula (5) is

$$
\begin{aligned}
P\left\{x_{k}<\frac{1}{m_{k}}\right\} & =P\left\{\frac{x_{k}-\mu}{\sigma_{k}}<\frac{m_{k}^{-1}-\mu}{\sigma_{k}}\right\} \\
& =P\left\{z_{k}<g\left(m_{k}\right)=\frac{m_{k}^{-1}-\mu}{\sigma_{k}}\right\} \\
& =\int_{-\infty}^{g\left(m_{k}\right)} f\left(z_{k}\right) d z_{k}=1-\alpha .
\end{aligned}
$$


Therefore,

$$
m_{k}=g^{-1}\left(F_{Z}^{-1}(1-\alpha)\right)=\left[\mu+\sigma_{k} F_{Z}^{-1}(1-\alpha)\right]^{-1},
$$

where $\sigma_{k}$ can be obtained from SV modeling estimation and $F_{Z}^{-1}(1-\alpha)$, high fractal of $f\left(z_{k}\right)$ under confidence level $1-$ $\alpha$, still needs to be estimated; $f\left(z_{k}\right)$ is probability density function of residual term $z_{k}$.

In the standard model, $z_{k}$ is independent of identically distributed white noise, following normal distribution. In the application of SV modeling, because of the fact that return on assets series does not follow normal distribution, we always assume that $z_{k}$ follows $t$ distribution, generalized error distribution (GED), and mixed normal distribution to describe the kurtosis, fat tail, and skewness of return on assets. We introduce extreme value theory (EVT) to describe the characters of $z_{k}$ from formula (7). We are interested in extreme risk of gap risks in CPPI strategy, that is, high score sites of loss distribution. We do not have to estimate the entire loss distribution because EVT can describe the tail of loss, accordingly avoid assumption of loss distribution, and reduce the risk of the model. Additionally, extreme value rarely occurs in real data, and estimate efficiency will be affected when estimating quantile by estimating distribution. However, EVT makes the extrapolation based on practice distribution smooth and thereby shows the entire shape of the tail rather than several losses in the tail. It suits the estimation under high confidence level involved in CPPI gap risk management.

Extreme value theory is used specifically for abnormal phenomena and small probability event. It is not modeled for the entire distribution but concentrates on the approximate expression of the tail distribution. Two types of model mainly include traditional block maxima and peaks over threshold (POT) model. POT model can use the original data more effectively when observed value exceeds a certain big enough threshold, especially for the financial risk measurement and modeling [15]. It is suitable for the description of extreme risk of gap risk in CPPI strategy. Thus, POT modeling was used to describe tail area of $z_{k}$ distribution.

$F(x)$ denotes cumulative distribution function (CDF) of variable $X ; x_{+}$is upper extreme point of $F . u$ denotes the big enough threshold value; the number of samples exceeding the threshold is $N_{u} ; x_{1}, \ldots, x_{N_{u}}$ denotes sample observations of exceeding samples; let $y=x-u$; the exceeding loss distribution $F_{u}(y)$ is shown in

$$
F_{u}(y)=P(X-u \leq y \mid X>u)=\frac{F(y+u)-F(u)}{1-F(u)} .
$$

$F_{u}(y)$ defines probability distribution of exceeding loss on the right end, which is also cumulative probability distribution of variable exceeding $u$ under the condition of $x>u$. According to Pickands III theorem [16], when $u \rightarrow x_{+}$, for a wide range probability distribution $F(x)$

$$
\lim _{u \rightarrow x+} \sup _{0 \leq y \leq x_{+}-u}\left|F_{u}(y)-G_{\xi, \beta(u)}(y)\right|=0
$$

holds for certain $\xi$ and $\beta(u)$. In other words, for a large enough threshold $u, F_{u}(y)$ tends to be generalized Pareto distribution $(\mathrm{GPD}) G_{\xi, \beta(u)}(\cdot)$. Its function is as follows:

$$
G_{\xi, \beta}(y)= \begin{cases}1-\left(1+\frac{\xi y}{\beta}\right)^{-1 / \xi} & \text { if } \xi \neq 0, \\ 1-\exp \left(-\frac{y}{\beta}\right) & \text { if } \xi=0 .\end{cases}
$$

$\xi$ denotes shape parameter; $\beta$ denotes scale parameter. After having the distribution function of exceeding loss, let $x=y+$ $u$; the tail distribution function is shown as

$$
F(x)=(1-F(u)) G_{\xi, \beta}(x-u)+F(u) .
$$

$N$ denotes the total number of observed samples; $N_{u}$ denotes the number of samples exceeding the threshold $u$. From empirical data, we get the estimated value of $F(u),(N-$ $\left.N_{u}\right) / N$, which means that when $x$ is large enough, the estimation of tail cumulative distribution is

$$
\begin{aligned}
F(x) & =\frac{N_{u}}{N}\left[1-\left(1+\frac{\xi}{\beta}(x-u)\right)^{-1 / \xi}\right]+\left(1-\frac{N_{u}}{N}\right) \\
& =1-\frac{N_{u}}{N}\left(1+\frac{\xi}{\beta}(x-u)\right)^{-1 / \xi} ;
\end{aligned}
$$

and thus,

$$
F_{Z}^{-1}(1-\alpha)=u+\frac{\beta}{\xi}\left[\left(\frac{N}{N_{u}} \alpha\right)^{-\xi}-1\right] .
$$

Putting it into formula (7), we get

$$
m_{k}=\frac{1}{\mu+\sigma_{k} u+\sigma_{k}(\beta / \xi)\left[\left(\left(N / N_{u}\right) \alpha\right)^{-\xi}-1\right]} .
$$

Formula (14) reveals the function relationship of multiple $m$ and risky assets value, frequency of extreme price, or expected loss under the requirement of gap risk management. When risky assets value fluctuated wildly, reduce the multiple to reduce the risk exposure; when $\xi$ is increasingly larger, corresponding multiple gets smaller and smaller. Parameter $\xi$ depends on distribution shape and denotes the heaviness of tail distribution. It indicates that when there is more frequent extreme price, the risk exposure of CPPI portfolio should be reduced. $\mu$ denotes the expected loss of risky assets. When expected loss is small, enlarge multiple to increase risk exposure. It is intuitive that the proportion of risky assets in CPPI portfolio should be increased in bull market, but formula (14) reveals that multiple setting should consider other factors from a gap risk management perspective, not to overincrease risk exposure in bull market.

\section{Empirical Studies}

This paper selects the Shanghai composite index as a risky asset investment portfolio (from January 02, 1997, to December 31, 2010), for the following reasons. (1) From 


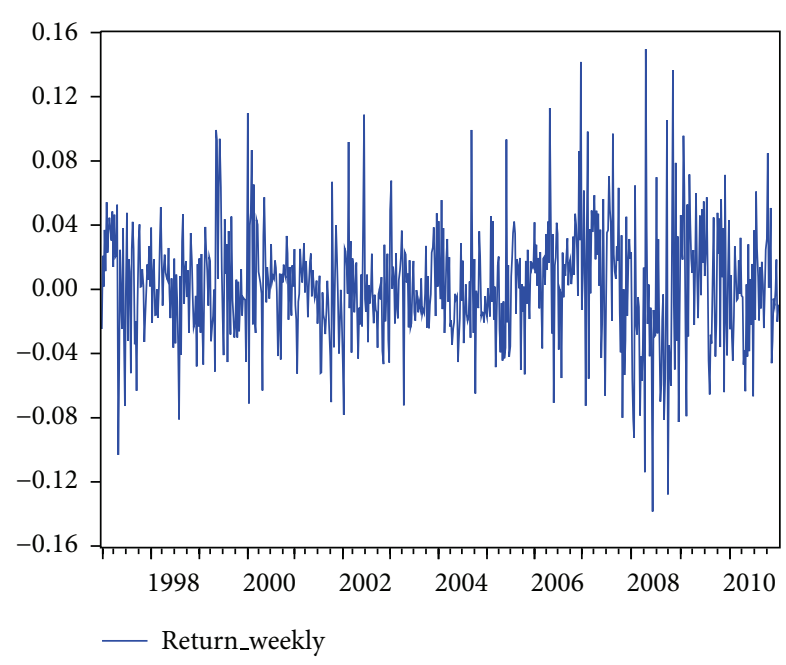

FIGURE 2: Time series of Shanghai composite index returns.

the perspective of risk measurement, the change of the Shanghai composite index can reflect system risk. Moreover, it covers the influence of extreme events such as Asian financial crisis that happened in 1997 and the United States financial crisis of 2008. (2) From the perspective of model estimation, the Shanghai composite index has been compiled for the longest time, which guarantees that there are enough samples, especially plenty of weekly return data. (3) From the perspective of the time span, because it was not until December 16, 1996, that the price limit regime began to be implemented, we choose the time span which is from January 1997 on so as to eliminate the abnormal samples resulting from the unsound regulations, such as a $105.27 \%$ boom in a single day on May 21, 1992. Corresponding to week by week fixed cycle adjustment principle, we analyze assets value changing by weekly return rate. 700 samples are included, with percentile return rate.

3.1. Data Description. Firstly, basic statistical description of return series is given. Figure 2 shows time series of Shanghai composite index returns, in which wave agglomeration effect can be seen in the series. Basic statistical description of return series can be seen in Table 1; $P$ values of corresponding statistics are shown in brackets. Compared with the normal distribution (skewness 0 and kurtosis 3), skewness and kurtosis characteristics can be shown in return series. Meanwhile, Jarque-Bera normality test also shows a remarkable difference from normal distribution. Moreover, ADF test of unit root shows stability of return series, and Ljung-Box test shows a noticeable autocorrelation. Thus, time series of Shanghai composite index returns shows the characteristics of kurtosis, skewness, and aggregation, which is suitable for SV modeling and analysis.

3.2. Results. Secondly, dynamic multiple is set by model parameter estimation in formula (14). It is achieved by estimating the SV model by loss series of Shanghai composite index returns. Fluctuation in SV model is a latent variable,
TABLE 1: Statistical characteristics of shanghai composite index returns.

\begin{tabular}{lc}
\hline Statistical indicators & Value \\
\hline Mean & 0.002 \\
Maximum & 0.149 \\
Minimum & -0.138 \\
Standard deviation & 0.035 \\
Skewness & 0.194 \\
Kurtosis & 4.570 \\
J-B statistic & $76.36(0.000)$ \\
ADF test & $-13.030(0.000)$ \\
L-B Q(5) & $14.006(0.016)$ \\
L-B Q(10) & $22.238(0.014)$ \\
\hline
\end{tabular}

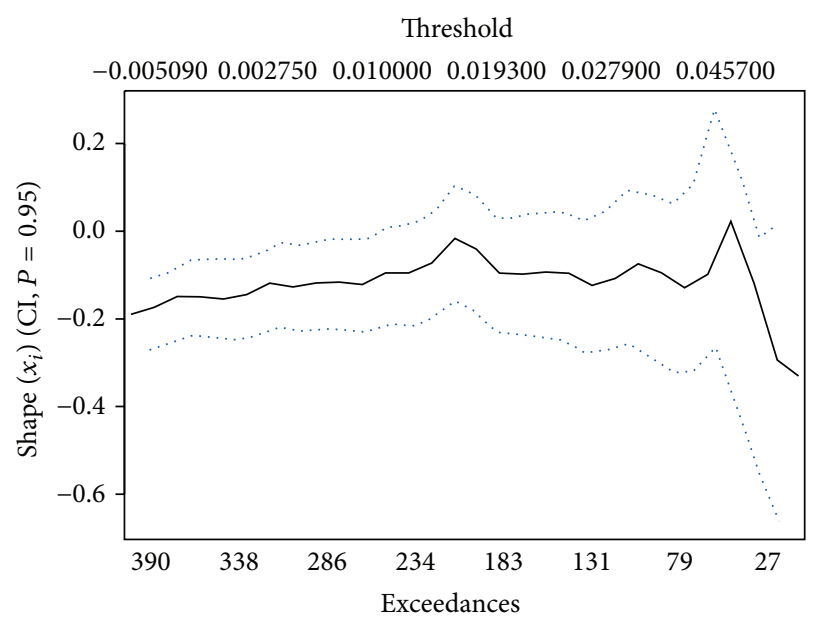

FIGURE 3: Estimates of shape parameter as a function of the threshold value.

and it is difficult to estimate the parameters by maximum likelihood method; thus, Bayesian principle and MCMC method are used in this paper [17]. By constructing a Markov chain for the sampling of given multivariate probability density, statistical inference is made with the help of ergodicity of Markov chain. Programmed and computed in Winbugs [18], the expected estimation values of Bayesian posterior distribution in SV modeling parameter are $\widehat{v}=0.007, \widehat{\phi}=$ 0.859 , and $\widehat{\tau}=0.119$.

After that we estimate high fractal $F_{Z}^{-1}(1-\alpha)$ of residual sequence $z_{k}$. First of all, we choose an appropriate threshold value estimate generalized Pareto distribution parameter. Since shape parameter $\xi$ is the limited index of distribution independent of threshold value $u$, an effective way to choose the threshold value (Figure 3 ) is to observe the shape parameter $\xi$ estimation curve in different threshold value. Generally, we choose threshold value when shape parameter $\xi$ is relatively stable.

$$
F(x)=\frac{N_{u}}{N}\left[1-\left(1+\frac{\xi}{\beta}(x-u)\right)^{-1 / \xi}\right]+\left(1-\frac{N_{u}}{N}\right) .
$$




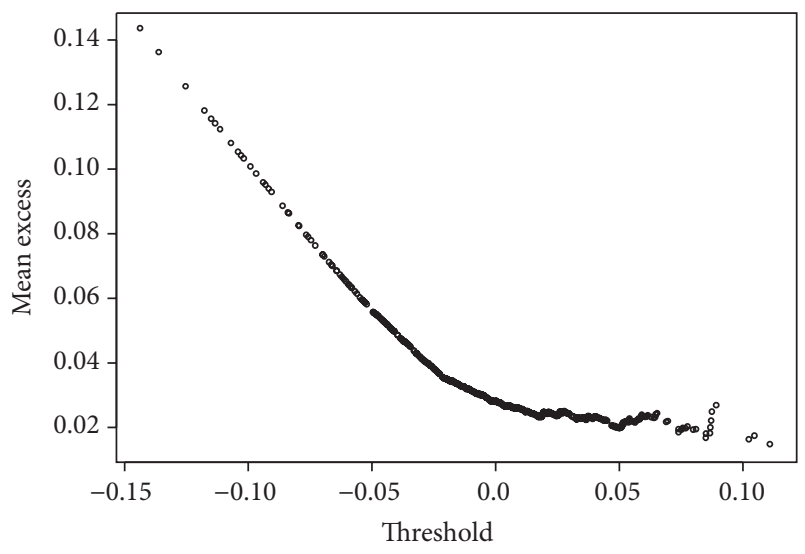

FIGURE 4: Empirical mean excess plot.

TABLE 2: Estimates of GPD parameters and quantile of residuals $z_{k}$.

\begin{tabular}{lcccc}
\hline$u$ & $\widehat{\xi}$ & $\widehat{\beta}$ & $N_{u} / N$ & $F_{Z}^{-1}(1-\alpha)$ \\
\hline 0.045 & -0.101 & 0.024 & $9.714 \%$ & 0.133 \\
\hline
\end{tabular}

Here, $u=0.045$. We can also confirm through empirical mean excess function plot (EMEF), which is the curve of points $\left(u, e_{N}(u)\right)$. The definition of $e_{N}(u)$ can be seen in formula (16). The basis for the selection is whether a linear trend is shown after exceeding a certain threshold. Figure 4 shows that threshold should be about 0.05 . Considering the judgment from Figures 3 and 4, we choose the threshold value $u=0.045$ :

$$
\begin{gathered}
e_{N}(u)=\frac{\sum_{i=a}^{N}\left(x_{i}-u\right)}{N-a-1}, \\
a=\min \left\{i \mid x_{i}>u\right\} .
\end{gathered}
$$

After $u$ is given, we can have estimated value of parameters $\xi$ and $\beta(u)$ based on maximum likelihood method estimation. For a given generalized Pareto distribution sample, $\left\{y_{1}, \ldots, y_{N_{u}}\right\}$, GPD log-likelihood function of GPD can be seen in formula (17). When $1-\alpha=99.9 \%$, calculate $F_{Z}^{-1}(1-\alpha)$; the results are shown in Table 2:

$$
\begin{aligned}
& L(\xi, \beta \mid y) \\
& \quad= \begin{cases}-N_{u} \ln \beta-\left(1+\frac{1}{\xi}\right) \sum_{i=1}^{N_{u}} \ln \left(1+\frac{\xi}{\beta} y_{i}\right) & \xi \neq 0, \\
-N_{u} \ln \beta-\frac{1}{\beta} \sum_{i=1}^{N_{u}} y_{i} & \xi=0 .\end{cases}
\end{aligned}
$$

Figure 5 shows the sensitivity of $99.9 \%$ quantile estimates to changes in the threshold. Obviously, when the threshold value is large enough, the estimated value of $F_{Z}^{-1}(1-\alpha)$ is not sensitive to the change of $u$. This indicates that the estimation result has strong stability. Putting $\sigma_{k}$ and parameter estimation results in Table 2 into formula (14), we have dynamic setting of multiple $m$ in CPPI strategy (see Figure 6). The selection range of multiple is about $5 \sim 10$,

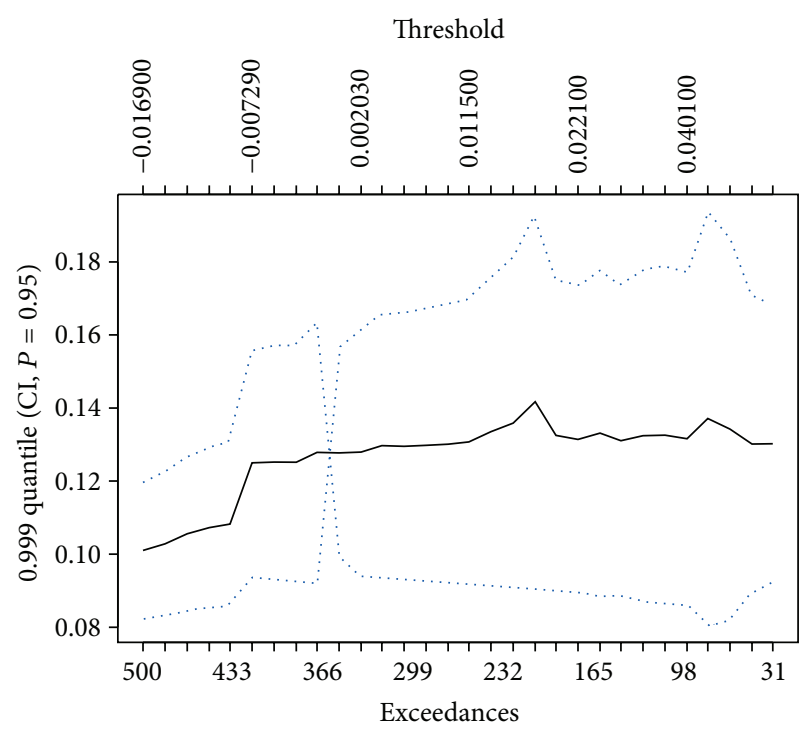

FIGURE 5: The sensitivity of $99.9 \%$ quantile estimates to changes in the threshold.

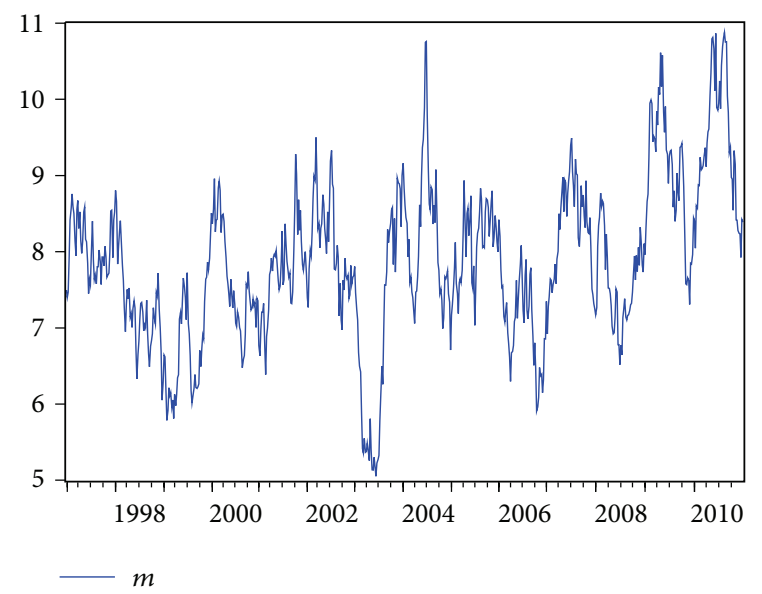

FIGURE 6: Determination of dynamic CPPI multiple.

and the mean and the median are both 7.8. This range is a little larger than the static multiple setting from Jessen [10]. Apart from different research methods, it is probably because dynamic multiple setting increased risky assets investment under the premise of gap risk avoiding, thus achieving a higher expected value.

\section{Conclusions}

From a perspective of gap risk management, this paper proposes a dynamic setting model of the parameter "Multiple" of CPPI strategy. According to the statistical estimation of extreme value change of risky asset by using SVEVT approach, a dynamic choice of multiple is detailed as a function of time-varying asset volatility, expected loss, and the possibility of occurrence of extreme events in the active asset returns. The empirical illustration on Shanghai 
composite index data shows that the multiple should be chosen dynamically in the interval 5 10, which is larger than the static results in prior studies. Our research promotes the applicability of CPPI strategy in emerging capital market by providing useful reference and tools for CPPI investors to manage gap risk and choose a proper risk exposure level. The model demonstrates the effect of time-varying asset volatility, expected loss, and the possibility of occurrence of extreme events in the active asset returns on multiple setting. Therefore, investors can make decisions on changing multiple to manage gap risk based on analyzing market conditions from the above-mentioned three dimensions. Moreover, the model allows investors to choose a moderate risk control level according to their specific management target and risk preference.

\section{Conflict of Interests}

The authors declare that there is no conflict of interests regarding the publication of this paper.

\section{Acknowledgment}

This work is supported by the National Natural Science Foundation of China (Project no. 71073124).

\section{References}

[1] F. Black and R. Jones, "Simplifying portfolio insurance for corporate pension plans," The Journal of Portfolio Management, vol. 14 , no. 4 , pp. 33-37, 1988 .

[2] G. Kingston, "Theoretical foundations of constant-proportion portfolio insurance," Economics Letters, vol. 29, no. 4, pp. 345347, 1989.

[3] F. Black and A. F. Perold, "Theory of constant proportion portfolio insurance," Journal of Economic Dynamics and Control, vol. 16, no. 3-4, pp. 403-426, 1992.

[4] H. P. Huang and Y. F. Tang, "The application of CPPI strategy in guaranteed fund," Management Review, vol. 18, no. 4, pp. 10-13, 2006.

[5] J. S. Chen, C. L. Chang, J. L. Hou, and Y. T. Lin, "Dynamic proportion portfolio insurance using genetic programming with principal component analysis," Expert Systems with Applications, vol. 35, no. 1-2, pp. 273-278, 2008.

[6] J. S. Chen and B. P. Liao, "Piecewise nonlinear goal-directed CPPI strategy," Expert Systems with Applications, vol. 33, no. 4, pp. 857-869, 2007.

[7] H. I. Lee, M. H. Chiang, and H. Hsu, "A new choice of dynamic asset management: the variable proportion portfolio insurance," Applied Economics, vol. 40, no. 16, pp. 2135-2146, 2008.

[8] S. Balder, M. Brandl, and A. Mahayni, "Effectiveness of CPPI strategies under discrete-time trading," Journal of Economic Dynamics \& Control, vol. 33, no. 1, pp. 204-220, 2009.

[9] R. Cont and P. Tankov, "Constant proportion portfolio insurance in the presence of jumps in asset prices," Mathematical Finance, vol. 19, no. 3, pp. 379-401, 2009.

[10] C. Jessen, "Constant proportion portfolio insurance: discretetime trading and gap risk coverage," in Proceedings of the 23rd Australasian Finance and Banking Conference, Sydney, Australia, December 2010.
[11] R. F. Engle, "Autoregressive conditional heteroscedasticity with estimates of the variance of United Kingdom inflation," Econometrica, vol. 50, no. 4, pp. 987-1007, 1982.

[12] T. Bollerslev, "Generalized autoregressive conditional heteroskedasticity," Journal of Econometrics, vol. 31, no. 3, pp. 307327, 1986

[13] S. J. Taylor, "Modeling stochastic volatility: a review and comparative study," Mathematical Finance, vol. 4, no. 2, pp. 183204, 1994.

[14] S. Kim, N. Shephard, and S. Chib, "Stochastic volatility: likelihood inference and comparison with ARCH models," The Review of Economic Studies, vol. 65, no. 3, pp. 361-393, 1998.

[15] A. J. McNeil and R. Frey, "Estimation of tail-related risk measures for heteroscedastic financial time series: an extreme value approach," Journal of Empirical Finance, vol. 7, no. 3-4, pp. 271-300, 2000.

[16] J. Pickands III, "Statistical inference using extreme order statistics," The Annals of Statistics, vol. 3, no. 1, pp. 119-131, 1975.

[17] S. Chib, F. Nardari, and N. Shephard, "Markov chain Monte Carlo methods for stochastic volatility models," Journal of Econometrics, vol. 108, no. 2, pp. 281-316, 2002.

[18] R. Meyer and J. Yu, "BUGS for a Bayesian analysis of stochastic volatility models," Econometrics Journal, vol. 3, no. 2, pp. 198$215,2000$. 


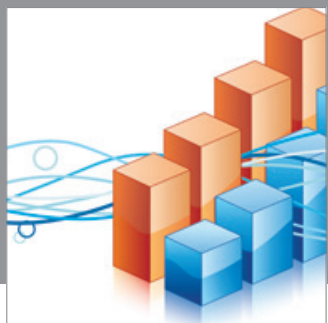

Advances in

Operations Research

mansans

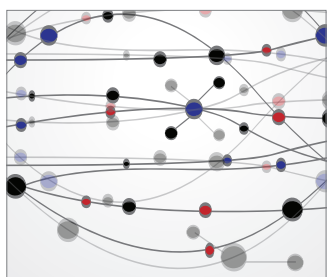

The Scientific World Journal
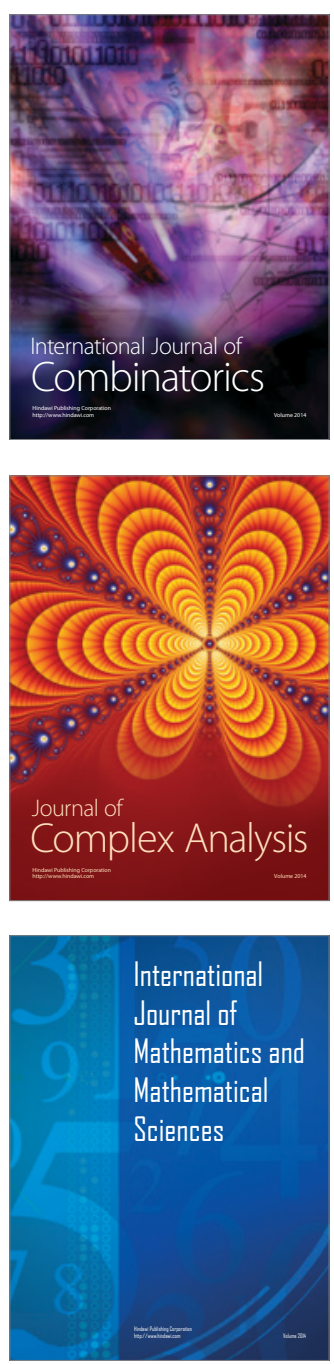
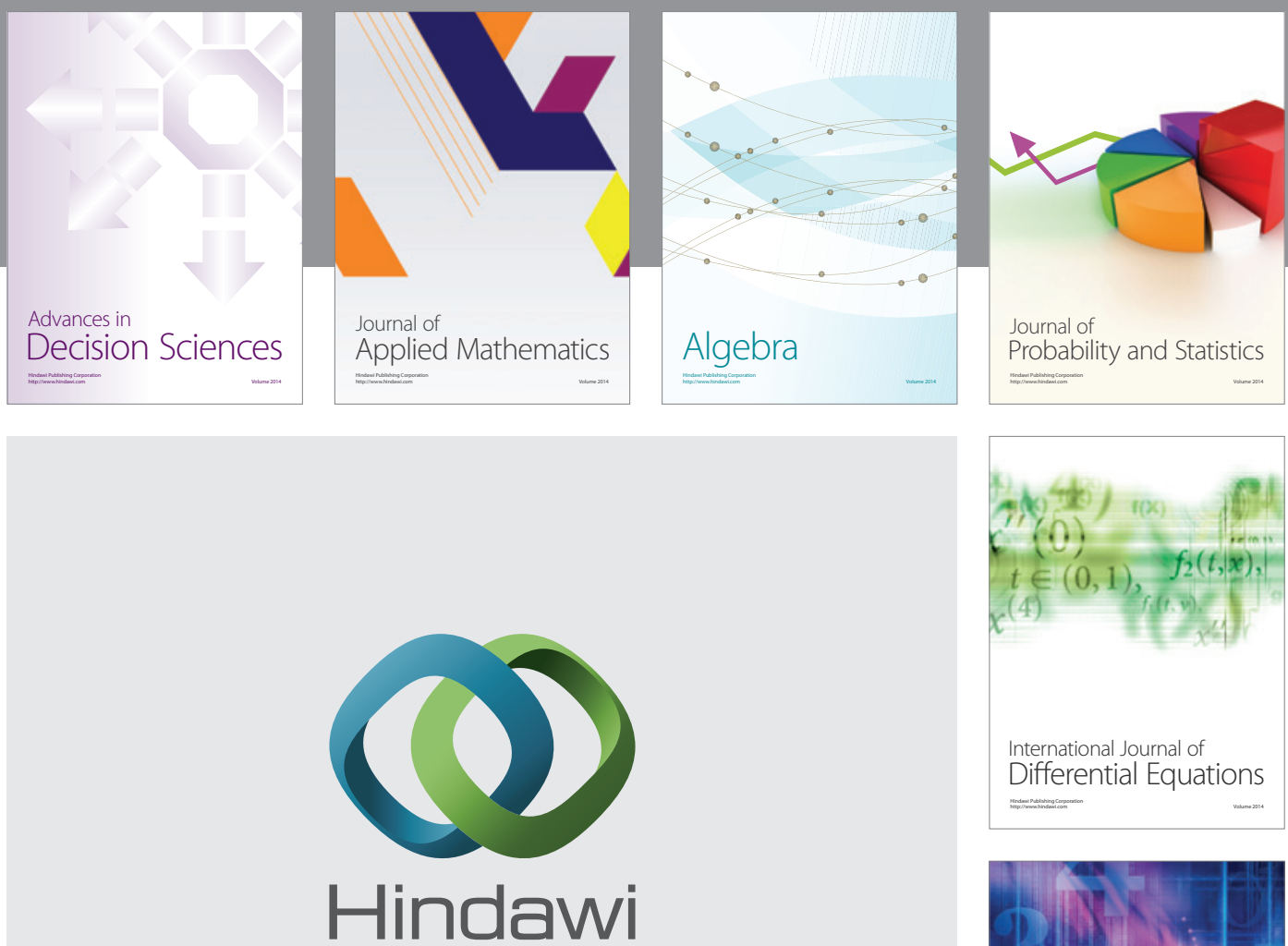

Submit your manuscripts at http://www.hindawi.com
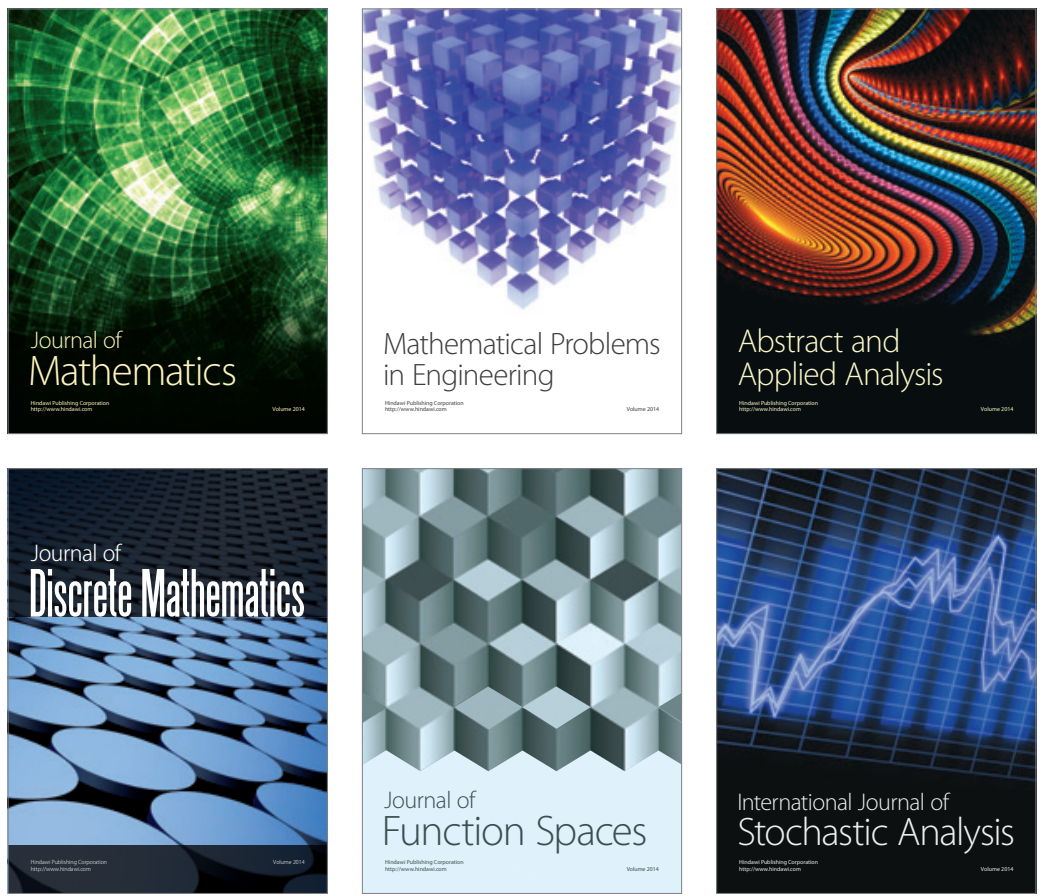

Journal of

Function Spaces

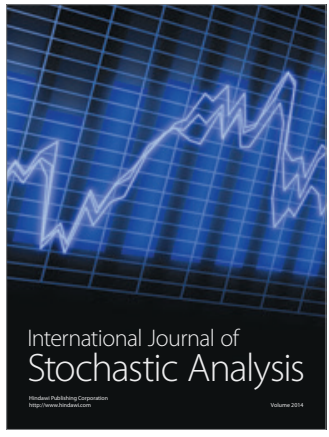

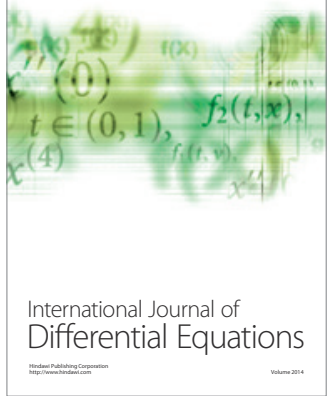
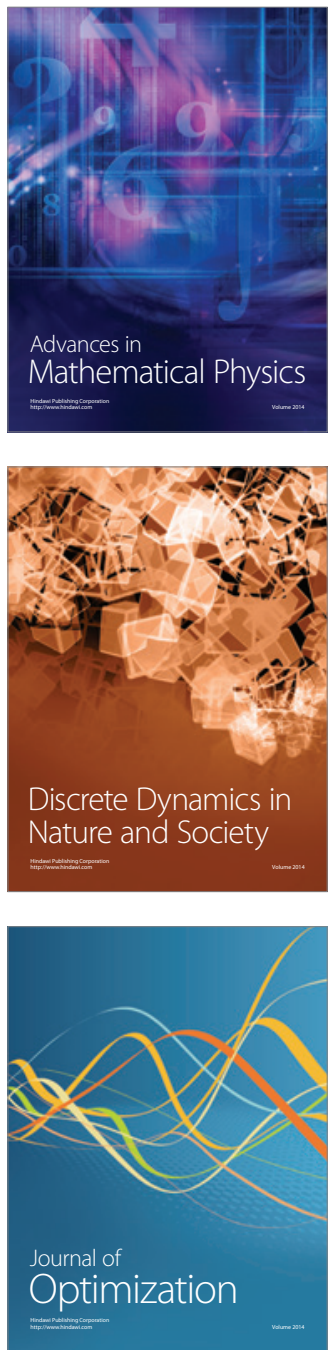